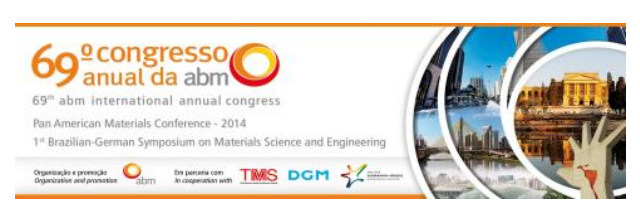

Tema: Tratamento Térmico

\title{
AVALIAÇÃO DO DESEMPENHO MECÂNICO DE UM AÇO MULTICONSTITUÍDO E REVENIDO COM ALTO TEOR DE Si ${ }^{\star}$
}

\author{
José Alberto da Cruz Junior ${ }^{1}$ \\ Renata Loures Lucinda ${ }^{2}$ \\ Isabela Viegas Aguiar ${ }^{2}$ \\ Dagoberto Brandão Santos ${ }^{3}$
}

\begin{abstract}
Resumo
Aços multiconstituídos têm aplicações voltadas principalmente para o setor automobilístico. Isso porque esses apresentam uma boa combinação de elevada resistência, ductilidade e tenacidade. A elevada resistência desses aços é devida a martensita e aos finos feixes de ferrita bainítica, enquanto que a tenacidade e a ductilidade são atribuídas aos filmes finos de austenita retida presentes entre os feixes. Esses filmes contém uma maior concentração de carbono em relação aos blocos de austenita retida. Uma consequência é que estes se tornam mais estáveis mecanicamente e termicamente. Dessa forma, foi realizada a avaliação do desempenho mecânico de um aço tratado isotermicamente abaixo de $M_{i}$ e revenidos a $400^{\circ} \mathrm{C} / 1 \mathrm{~h}$. Os resultados dos ensaios de impacto mostraram que quanto maior a fração volumétrica da austenita retida maior é a energia absorvida, independente da morfologia desse constituinte.
\end{abstract}

Palavras-chave: Martensita; Bainita; Austenita retida; Revenimento.

\section{EVALUATION OF MECHANICAL PERFORMACE OF THE MULTI-PHASE STEEL AND TEMPERED WITH HIGH CONTENT OF Si}

\section{Abstract}

Multi-phase steels have applications primarily focused on the automobile industry. This is because these have a good combination of high strength, ductility and toughness. The high strength of these steels is due to martensite and thin plates of bainitic ferrite, while the toughness and ductility are attributed to thin films of retained austenite present between the plates. These films contain a higher concentration of carbon compared to blocks of retained austenite. The consequence is that these become more mechanically and thermally stable. Thus, the evaluation of the mechanical performance of a treated steel isothermally below $\mathrm{M}_{\mathrm{s}}$ and tempered at $400^{\circ} \mathrm{C} / 1 \mathrm{~h}$ was performed. The results of the impact tests showed that the higher the volume fraction of retained austenite the greater is absorbed energy, independent of the morphology of this constituent.

Keywords: Martensite; Bainite; Retained austenite; Tempering.

1 Graduando em Engenharia de Materiais, CEFET, aluno do Programa de Pós-Graduação em Engenharia Metalúrgica, Materiais e de Minas, UFMG, Associado da ABM, Belo Horizonte, MG, Brasil.

2 Graduanda em Engenharia Metalúrgica e de Materiais, UFMG, Belo Horizonte, MG, Brasil.

3 Professor Associado, Departamento de Engenharia Metalúrgica e de Materiais, UFMG, Membro da ABM, Belo Horizonte, MG, Brasil.

\footnotetext{
* Contribuição técnica ao $69^{\circ}$ Congresso Anual da ABM - Internacional e ao 14ํㅡㄹ ENEMET - Encontro Nacional de Estudantes de Engenharia Metalúrgica, de Materiais e de Minas, 21 a 25 de julho de 2014, São Paulo, SP, Brasil.
} 


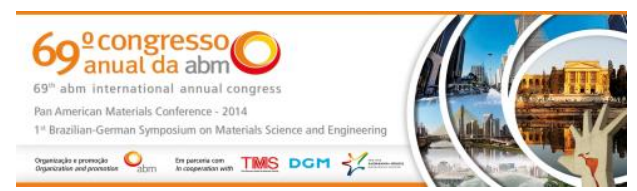

\section{INTRODUÇÃO}

A convencional microestrutura bainítica é classificada como bainita superior e inferior. Essas são compostas de placas de ferrita bainítica separadas por camadas contínuas de cementita. A principal diferença é que partículas de cementita precipitam também no interior das placas de ferrita, como é o caso da bainita inferior [1]. Isso ocorre porque a menor difusão do carbono associada com a diminuição da temperatura leva-o a se precipitar como finos carbonetos na ferrita bainítica [2,3]. Por causa da espessa camada de cementita, aços bainíticos não apresentam na prática o mesmo desempenho mecânico que os aços martensíticos temperados e revenidos [4]. No entanto, se uma quantidade suficiente de elementos de liga $(1,5 \%$ em peso de Si) é adicionada, este retarda a precipitação de cementita. O silício tem baixa solubilidade na cementita, ou seja, o silício, que é rejeitado, aumenta a sua concentração em torno dos embriões de cementita. Assim, os átomos de Si funcionam como uma barreira no entorno dos embriões de cementita, reduzindo o fluxo de carbono e, consequentemente, inibindo o seu crescimento [5-7]. A microestrutura do aço com alto teor de $\mathrm{Si}$, transformado em bainita a baixa temperatura, é composta de finas placas de ferrita bainítica separadas por regiões de austenita retida enriquecida em carbono e ainda pode conter alguma martensita [8-11].

A baixa temperatura de transformação favorece uma maior quantidade de ferrita bainítica que por sua vez diminui a fração de blocos de austenita retida, melhorando suas propriedades mecânicas [12-15]. Os filmes de austenita retida contém uma maior concentração de carbono do que os blocos, devido ao excesso de carbono presente que se difunde da ferrita bainítica para os filmes. Esta austenita retida torna-se mais estável termicamente e mecanicamente [16-18].

O resultado da baixa temperatura de transformação é que os feixes de ferrita bainítica são extremamente finos, $20-40 \mathrm{~nm}$ de espessura, tornando o material muito resistente. Valores de dureza de aproximadamente $600 \mathrm{HV}$, limite de resistência acima de $2,5 \mathrm{GPa}$ e de tenacidade a fratura de aproximadamente $130 \mathrm{MPa} \cdot \mathrm{m}^{1 / 2}$ foram obtidos em uma microestrutura nanoestruturada. Esses valores correspondem às propriedades mecânicas críticas dos aços marageing, o qual apresenta um custo de fabricação 30 vezes maior, por causa do alto teor de cobalto e níquel.

As diferentes frações volumétricas de ferrita bainítica e austenita retida leva a uma grande variação das propriedades mecânicas na qual tem suas aplicações voltadas principalmente para o setor automobilístico [19]. Existem outras aplicações que estão em pleno desenvolvimento como: eixos [20], aços resistentes ao desgaste [21] e componentes sinterizados [22].

Dessa forma, o presente estudo investigou a influência da temperatura de transformação, a fração volumétrica de austenita retida sobre a variação das propriedades mecânicas do aço comercial SAE 9254.

\section{MATERIAIS E MÉTODOS}

O aço usado nesse trabalho foi fornecido pela ArcelorMittal Aços Longos (João Monlevade/MG) e corresponde ao aço SAE 9254, utilizado para a fabricação de eixos, molas helicoidais, ferramentas, hastes para amortecedores, etc. Sua composição química é descrita na tabela 1. Nota-se o alto teor de Si adicionado de modo a evitar a precipitação de carbonetos [5-7] e a presença de $\mathrm{Mn}$ e Cr, a qual

\footnotetext{
* Contribuição técnica ao $69^{\circ}$ Congresso Anual da ABM - Internacional e ao 14ํㅡㄹ ENEMET - Encontro Nacional de Estudantes de Engenharia Metalúrgica, de Materiais e de Minas, 21 a 25 de julho de 2014, São Paulo, SP, Brasil.
} 


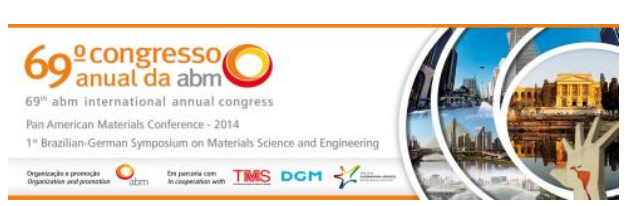

favorece a temperabilidade e inibe a formação de constituintes indesejáveis como a ferrita e perlita durante a têmpera.

$\mathrm{O}$ aço recebido no estado laminado a quente apresenta uma microestrutura de perlita fina e algumas ilhas de ferrita pró-eutetóide. Amostras foram usinadas como corpos de prova de tração e de impacto conforme as normas ASTM $[23,24]$ a partir do aço fornecido como fio-máquina com diâmetros de 8 e $20 \mathrm{~mm}$, respectivamente. Amostras foram austenitizadas a $900^{\circ} \mathrm{C}$ por 5 min e em seguida tratadas isotermicamente a 200,220 e $270^{\circ} \mathrm{C}$ por 0,5 e $48 \mathrm{~h}$. $\mathrm{O}$ tratamento isotérmico foi realizado em um banho térmico que consiste de uma liga $\mathrm{Sn60-Pb40}$ cujo ponto de fusão está abaixo de $200^{\circ} \mathrm{C}$. A temperatura foi controlada por um termopar tipo $\mathrm{K}$ posicionado junto às amostras. Após o tratamento isotérmico, as amostras foram resfriadas ao ar até a temperatura ambiente (Fig. 1). Nota-se na Figura 1 que as três temperaturas de tratamento isotérmico $\left(200,220,270^{\circ} \mathrm{C}\right)$ selecionadas estão abaixo da temperatura de início de transformação martensítica $\left(\mathrm{Mi}_{\mathrm{i}}\right.$ o que implica que uma dada fração volumétrica de martensita se formou no resfriamento inicial. Posteriormente, o restante da austenita residual se decompôs em bainita. Desta forma foi proposta a formação de bainita abaixo de $M_{i}$ de acordo com trabalhos prévios [25-27].

Tabela 1. Composição química em \%peso

\begin{tabular}{c|c|c|c|c|c|c}
\hline Aço & $\mathrm{C}$ & $\mathrm{Mn}$ & $\mathrm{Si}$ & $\mathrm{Cr}$ & $\mathrm{Ac}_{1}\left({ }^{\circ} \mathrm{C}\right)$ & $\mathrm{Ac}_{3}\left({ }^{\circ} \mathrm{C}\right)$ \\
\hline $\mathrm{C}-\mathrm{Si}$ & 0,54 & 0,58 & 1,43 & 0,47 & 766 & 820 \\
\hline
\end{tabular}

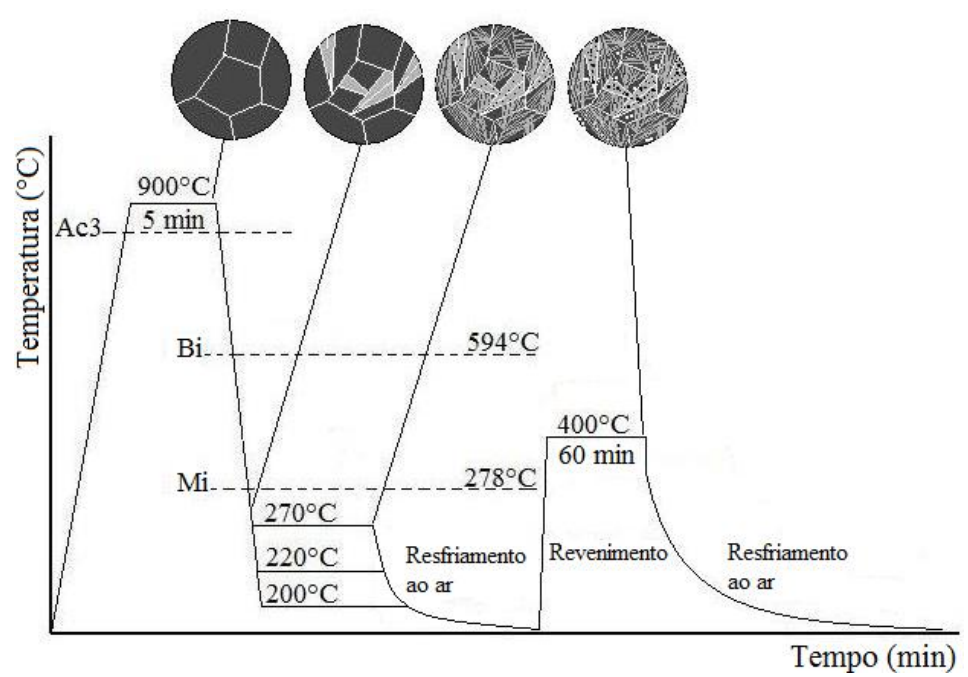

Figura 1. Representação esquemática do tratamento térmico visando à obtenção dos aços com estrutura multiconstituída de martensita, bainita e austenita retida.

As temperaturas usadas para os tratamentos isotérmicos foram escolhidas de forma a obter uma microestrutura multiconstituída. As temperaturas de início de formação da martensita e bainita foram, respectivamente, 278 e $594^{\circ} \mathrm{C}$, calculadas de acordo com as equações 1 e 2 [28]. Outras amostras além de serem tratadas isotermicamente nas mesmas condições foram também revenidas a $400^{\circ} \mathrm{C}$ por $1 \mathrm{~h}$ de modo a comparar sua microestrutura e as propriedades mecânicas com as amostras tratadas isotermicamente. Os resultados de um trabalho anterior [29] mostram que grande parte da austenita retida presente foi decomposta a $400^{\circ} \mathrm{C}$. Além disso, a escolha dessa temperatura se deve ao fato que esse aço é

\footnotetext{
* Contribuição técnica ao $69^{\circ}$ Congresso Anual da ABM - Internacional e ao 14ํㅡㄹ ENEMET - Encontro Nacional de Estudantes de Engenharia Metalúrgica, de Materiais e de Minas, 21 a 25 de julho de 2014, São Paulo, SP, Brasil.
} 


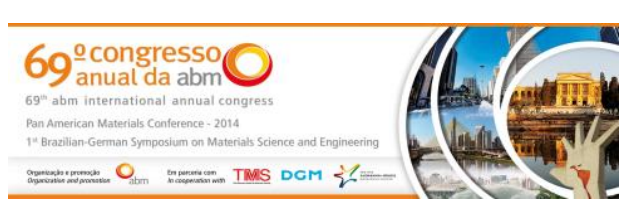

comercializado na condição temperado e revenido, sendo a temperatura de revenimento a mesma utilizada nesse trabalho.

$$
\begin{array}{r}
\mathrm{B}_{\mathrm{i}}\left({ }^{\circ} \mathrm{C}\right)=830-270 \mathrm{C}-90 \mathrm{Mn}-37 \mathrm{Ni}-70 \mathrm{Cr}-83 \mathrm{Mo} \\
\mathrm{M}_{\mathrm{i}}\left({ }^{\circ} \mathrm{C}\right)=539-423 \mathrm{C}-30,4 \mathrm{Mn}-17,7 \mathrm{Ni}-12,1 \mathrm{Cr}-7,5 \mathrm{Mo}
\end{array}
$$

$\mathrm{C}, \mathrm{Mn}, \mathrm{Cr}$, etc. são os elementos de liga (\%peso).

De acordo com a figura 1, para os tratamentos isotérmicos executados abaixo de $\mathrm{Mi}$, certa quantidade de martensita se formou no resfriamento inicial, essa pode ser estimada pela aplicação da equação (3) de Koistinen e Marburger [30].

$$
1-\mathrm{V}_{\alpha^{\prime}}=\exp \left[-0,011\left(\mathrm{M}_{\mathrm{i}}-\mathrm{T}_{\mathrm{i}}\right)\right]
$$

onde $\mathrm{Mi}_{\mathrm{i}}$ e $\mathrm{T}_{\mathrm{i}}$ representam as temperaturas de início de formação da martensita e de tratamento isotérmico, respectivamente. Essa martensita é revenida durante o tratamento isotérmico.

As amostras para metalografia foram preparadas pelo método convencional. 0 ataque químico foi realizado com o reativo Nital $2 \%$. A análise microestrutural foi realizada através da microscopia eletrônica de varredura (MEV). Amostras para análises em microscopia eletrônica de transmissão (MET) foram cortadas na forma de disco com $3 \mathrm{~mm}$ de diâmetro e lixadas em lixas (SiC) de granulometria de 1200 até a redução de $50 \mu \mathrm{m}$ de espessura final. Essas lâminas finas foram finalmente eletropolidas a baixa temperatura $\left(-30^{\circ} \mathrm{C}\right)$ até ocorrer um furo vazante. Para isso, foi utilizada a máquina Tenupol-5 operada a $40 \mathrm{~V}$. O eletrólito usado para realizar o furo consistiu em uma mistura de $80 \%$ de metanol, $15 \%$ de glicerol e $5 \%$ de ácido perclórico. Preparada as amostras, as mesmas foram levadas ao MET marca FEITecnai modelo G2-20 (200 kV) para uma melhor identificação dos constituintes e a verificação dos possíveis carbonetos presentes na microestrutura.

Foram realizadas análises de difração de elétrons retroespalhados (EBSD). Para isso, amostras dos corpos de prova Charpy tratadas isotermicamente por $0,5 \mathrm{~h}$ a 200,220 e $270^{\circ} \mathrm{C}$ foram polidas em uma politriz automática por $1 \mathrm{~h}$ em sílica coloidal de $0,04 \mu \mathrm{m}$ e os dados foram analisadas no software OIM através de mapas e texturas. Foi usado um MEV com filamento de $\mathrm{W}$ com potencial de aceleração de 20 $\mathrm{kV}$ e um passe de varredura de 0,5 a $3 \mu \mathrm{m}$.

Testes de microdureza foram executados com um equipamento de dureza Future Tech, equipado com o indentador Vickers com uma carga de 2,94 N (300 g) e um tempo de aplicação de carga de $20 \mathrm{~s}$. Vinte medidas foram executadas de maneira aleatória nas amostras.

Após o lixamento e polimento com pasta de diamante de $1 \mu \mathrm{m}$, as difrações foram executadas em um difratômetro Philips PW 1710, usando a radiação $\mathrm{K}_{\alpha}$ do $\mathrm{Cu}$ com uma taxa de $0,02 \% \mathrm{~min}$, tensão de $45 \mathrm{kV}$ e corrente de $45 \mathrm{~mA}$. Os dados da difração de raios-X foram também usados para determinar a concentração de carbono na austenita retida conforme a equação 3 . A fração volumétrica de austenita retida foi quantificada, usando o método da comparação direta. Este método usa a integração dos picos mais intensos da austenita, caracterizados pelos planos (111), (200), (211) e (220).

$$
a_{\mathrm{Y}}=3,578+0,033 \% \mathrm{C}_{\mathrm{Y}}
$$

$\mathrm{C}_{\gamma}$ é a concentração de carbono da austenita retida em \%peso e $\mathrm{a}_{\gamma}$ é o parâmetro de rede em $\AA[31,32]$.

\footnotetext{
* Contribuição técnica ao $69^{\circ}$ Congresso Anual da ABM - Internacional e ao 14ํㅡㄹ ENEMET - Encontro Nacional de Estudantes de Engenharia Metalúrgica, de Materiais e de Minas, 21 a 25 de julho de 2014, São Paulo, SP, Brasil.
} 


\section{RESULTADOS E DISCUSSÃO}

A figura 2 ilustra as micrografias das amostras tratadas isotermicamente a $200^{\circ} \mathrm{C}$, $220^{\circ} \mathrm{C}$ e $270^{\circ} \mathrm{C}$, e posteriormente submetidas ao revenimento. Todas as amostras apresentaram uma microestrutura constituída de martensita $\left(\alpha^{\prime}\right)$, ferrita bainítica $\left(\alpha_{b}\right)$ e austenita retida $\left(\mathrm{\gamma}_{\mathrm{r}}\right)$. No entanto, quanto menor à temperatura de tratamento isotérmico, menor a fração volumétrica de bainita. Isto ocorre porque há menos austenita para se transformar uma vez que certa quantidade de martensita se formou durante o resfriamento a partir de $900^{\circ} \mathrm{C}$ para a temperatura de tratamento isotérmico. Para as amostras posteriormente revenidas, certa quantidade de cementita precipitou-se a partir da austenita retida $\left(\mathrm{V}_{\mathrm{r}}\right)$. De acordo com trabalhos anteriores [13,33], a precipitação de cementita levou a uma diminuição do teor de carbono da austenita retida e consequentemente a formação de uma martensita não revenida, após o resfriamento até à temperatura ambiente. Além disso, as Figuras $2 \mathrm{~d}$ a $2 \mathrm{f}$ mostram que a decomposição da austenita retida ocorre principalmente nos blocos $\left(\gamma_{b}\right)$ (setas vermelhas), isso porque os filmes de austenita retida $\left(\gamma_{f}\right)$ são mais estáveis termicamente devido ao maior teor de carbono em relação aos blocos de austenita retida [6].

A figura 3 apresenta os mapas de orientação e a presença de duas possíveis fases cristalográfica: FCC para austenita retida e BCC para a ferrita, que é de fato bainita. Algumas regiões contendo martensita têm sido também indexadas como BCC, mas esta é associada com uma baixa qualidade de indexação do padrão de difração provavelmente devido a elevada densidade de deslocações na qual induz uma menor qualidade de reconhecimento do padrão indexado pelo sistema automático [34], conforme pode ser observado na figura 3. Devido à baixa fração volumétrica de austenita retida e a resolução espacial do equipamento, foi impossível a visualização da mesma nos mapas feitos com 10000x de ampliação. A figura 4 apresenta os resultados de textura determinado por EBSD a partir da Fig. 3 para as amostras tratadas isotermicamente a $270^{\circ} \mathrm{C} / 0,5 \mathrm{~h} \mathrm{Fig.} 4(\mathrm{a})$ e $220^{\circ} \mathrm{C} / 0,5 \mathrm{~h}$ Fig. 4 (b). A presença das componentes Goss, Latão e Cobre na austenita retida são típicos de textura de transformação [35].

Através das imagens de microscopia eletrônica de transmissão (MET) foi possível observar a presença de bainita inferior em uma amostra tratada isotermicamente a $200^{\circ} \mathrm{C} / 0,5 \mathrm{~h}$ e revenida a $400^{\circ} \mathrm{C} / 1 \mathrm{~h}$ (Fig. $5 \mathrm{a}$ ). A região em destaque na figura 3a ilustra a distribuição de carbonetos no interior da placa da bainita inferior. De acordo com Bhadeshia [36,37], os carbonetos usualmente se precipitam em uma única orientação cristalográfica, de tal modo que eles formam arranjos paralelos com aproximadamente $60^{\circ}$ em relação ao eixo da placa. Já para a amostra tratada isotermicamente a $270^{\circ} \mathrm{C} / 0,5 \mathrm{~h}$ e revenida $400^{\circ} \mathrm{C} / 1 \mathrm{~h}$ podem-se observar filmes de austenita retida intercalados com os feixes de ferrita bainítica, Figura $5 \mathrm{~b}$.

\footnotetext{
* Contribuição técnica ao $69^{\circ}$ Congresso Anual da ABM - Internacional e ao 14ํㅡㄹ ENEMET - Encontro Nacional de Estudantes de Engenharia Metalúrgica, de Materiais e de Minas, 21 a 25 de julho de 2014, São Paulo, SP, Brasil.
} 

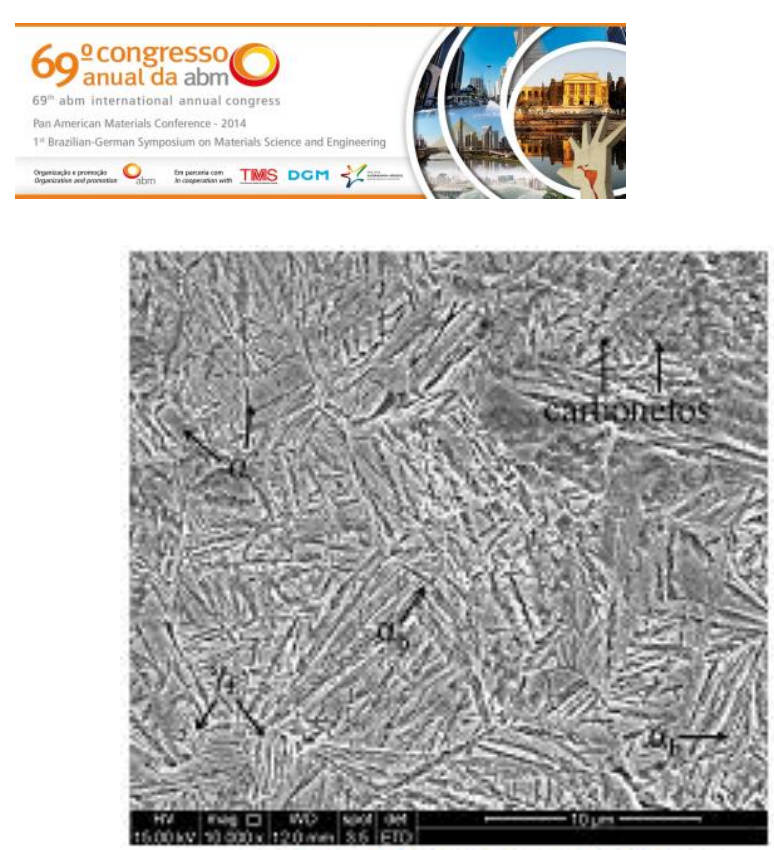

(a) tratada a $200^{\circ} \mathrm{C}$ por $0,5 \mathrm{~h}$.

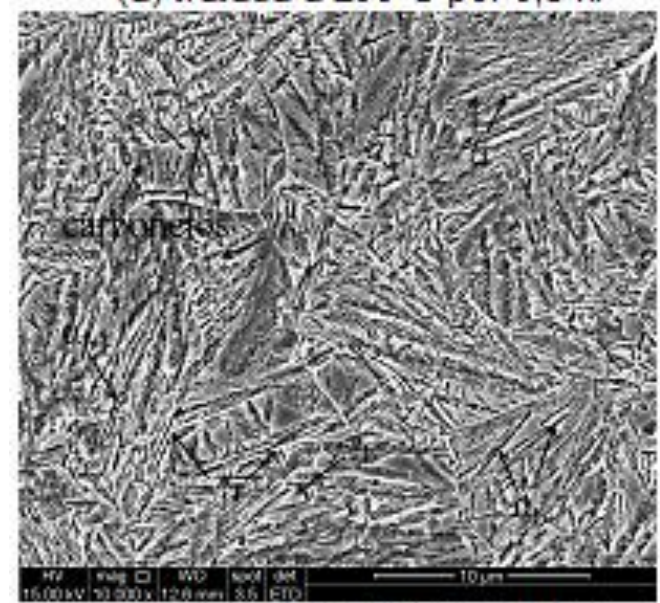

(b) tratada a $220^{\circ} \mathrm{C}$ por $0,5 \mathrm{~h}$

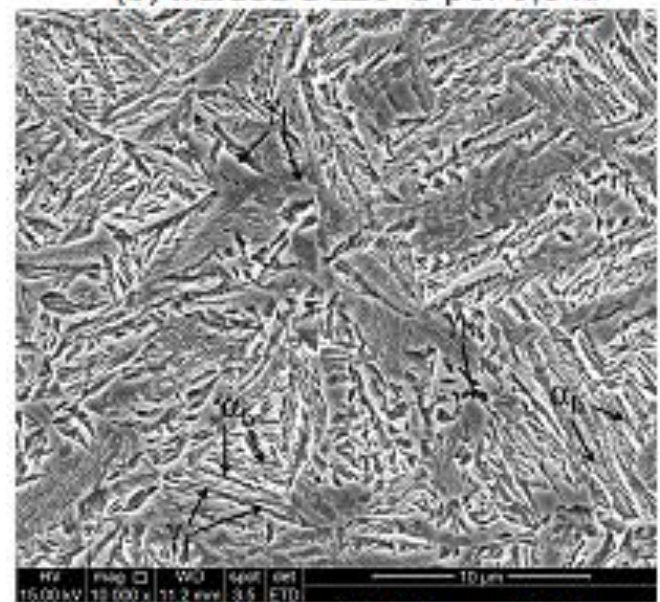

(c) tratada a $270^{\circ} \mathrm{C}$ por $0,5 \mathrm{~h}$.

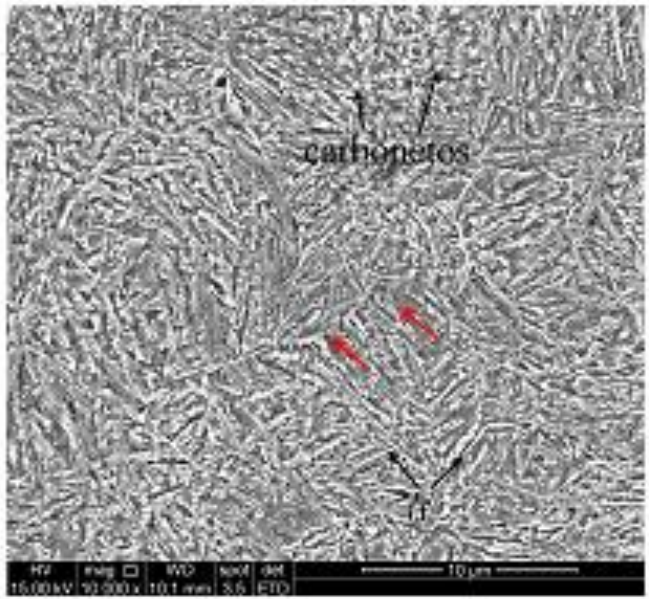

(d) idem (a), revenida a $400^{\circ} \mathrm{C}$ por $1 \mathrm{~h}$

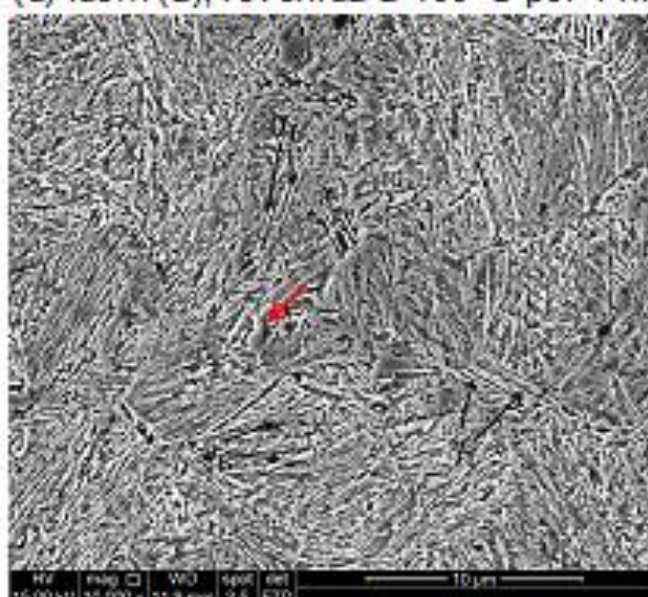

(e) idem (b), revenida a $400^{\circ} \mathrm{C}$ por $1 \mathrm{~h}$

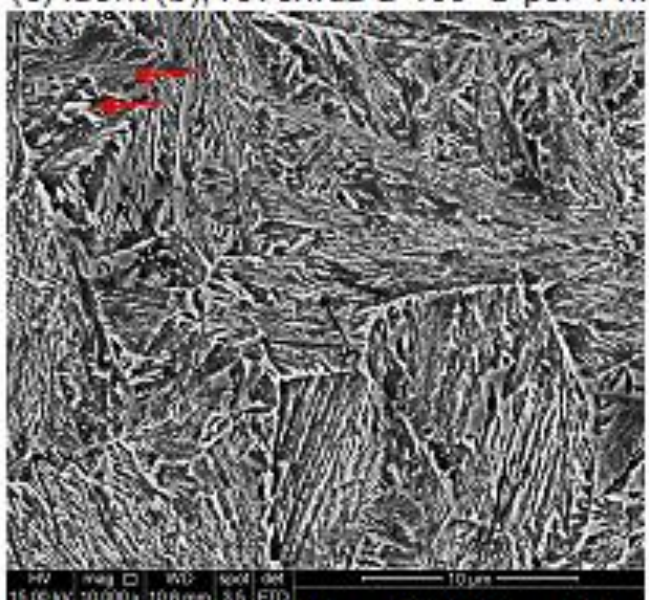

(f) idem (c), revenida a $400^{\circ} \mathrm{C}$ por $1 \mathrm{~h}$.

Figura 2. Microscopia eletrônica de varredura das amostras após a austenitização a $900^{\circ} \mathrm{C}$ e tratada isotermicamente a (a) 200; (b) 220 ; (c) $270^{\circ} \mathrm{C}$ por $0,5 \mathrm{~h}$. As figuras (d), (e) e (f) representam as mesmas amostras, respectivamente, no entanto revenidas a $400^{\circ} \mathrm{C}$ por $1 \mathrm{~h}$. As amostras foram atacadas com o reativo nital $2 \%$.

\footnotetext{
* Contribuição técnica ao 69 Congresso Anual da ABM - Internacional e ao 14 ENEMET - Encontro Nacional de Estudantes de Engenharia Metalúrgica, de Materiais e de Minas, 21 a 25 de julho de 2014, São Paulo, SP, Brasil.
} 
dureza, tal como a martensita, contribui para uma baixa tenacidade, conforme investigado nos trabalhos anteriores [8,9]. Isto está de acordo com o presente trabalho, uma vez que para a menor temperatura de tratamento isotérmico $\left(200^{\circ} \mathrm{C}\right)$ menor é a energia de impacto, devido a uma maior quantidade de martensita formada no resfriamento inicial.

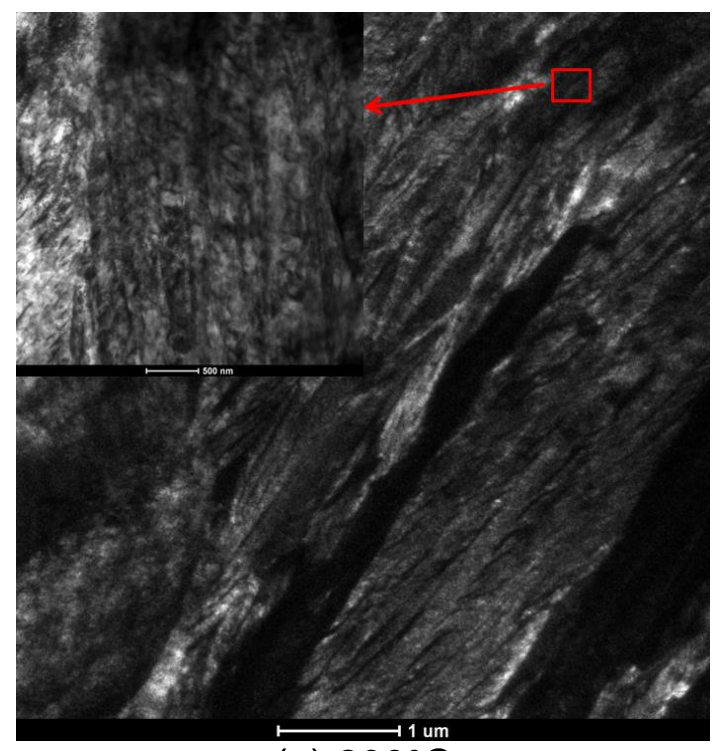

(a) $200^{\circ} \mathrm{C}$

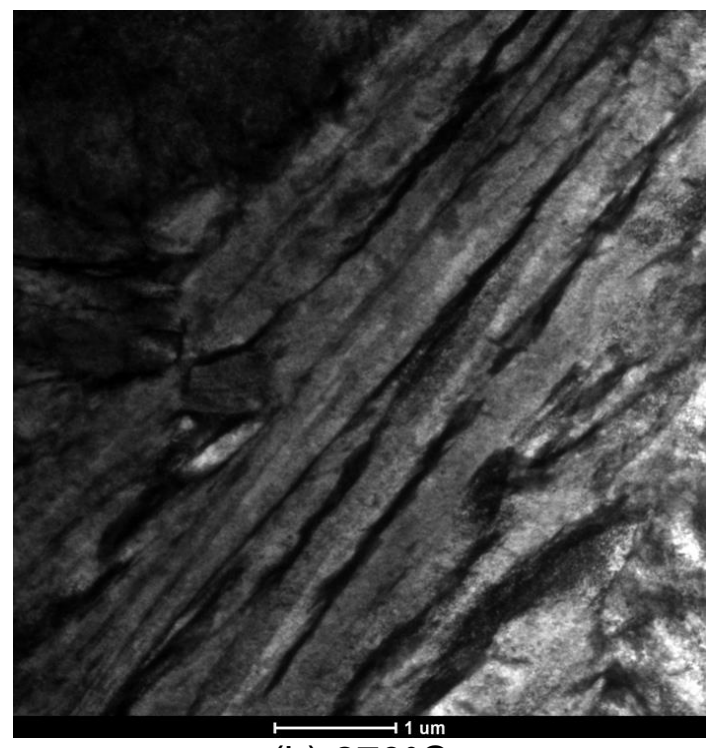

(b) $270^{\circ} \mathrm{C}$

Figura 5. Microscopia eletrônica de transmissão após transformação isotérmica a 200 e $270^{\circ} \mathrm{C}$ por $0,5 \mathrm{~h}$ e revenidas a $400^{\circ} \mathrm{C}$ por $1 \mathrm{~h}$.

Tabela 2. Fração volumétrica, conteúdo de carbono $\left(\mathrm{C}_{\mathrm{\gamma}}\right)$ da austenita retida $\left(\gamma_{\mathrm{r}}\right)$ e resultados das propriedades mecânicas: Limite de escoamento (LE) e de resistência (LR); Alongamento total (AT); Energia absorvida (EA)

\begin{tabular}{c|c|c|c|c|c|c|c}
\hline Tratamento isotérmico $\left({ }^{\circ} \mathrm{C} / \mathrm{h}\right)$ & $\begin{array}{c}\gamma_{\mathrm{r}} \\
(\%)\end{array}$ & $\begin{array}{c}\mathrm{C}_{\mathrm{V}} \\
(\%)\end{array}$ & $\begin{array}{c}\alpha^{\prime} \\
(\%)\end{array}$ & $\begin{array}{c}\mathrm{LE} \\
(\mathrm{MPa})\end{array}$ & $\begin{array}{c}\mathrm{LR} \\
(\mathrm{MPa})\end{array}$ & $\begin{array}{c}\mathrm{EA} \\
(\mathrm{J})\end{array}$ & $\begin{array}{c}\mathrm{AT} \\
(\%)\end{array}$ \\
\hline $200 / 0,5$ & 13,4 & 1,3 & 57,5 & 1092 & 1994 & 15,1 & 4,2 \\
\hline $200 / 0,5$ e revenida $400^{\circ} \mathrm{C} / 1 \mathrm{~h}$ & $5,4^{*}$ & $1,0^{*}$ & 57,5 & 1692 & 1778 & 11,0 & 6,8 \\
\hline $200 / 48$ & 8,2 & 1,0 & 57,5 & 1651 & 2105 & 14,8 & 2,3 \\
\hline $200 / 48$ e revenida $400^{\circ} \mathrm{C} / 1 \mathrm{~h}$ & $2,7^{*}$ & $0,7^{*}$ & 57,5 & 1552 & 1685 & 10,0 & 7,2 \\
\hline $220 / 0,5$ & 14,2 & 1,0 & 47,1 & 1217 & 2020 & 17,1 & 4,1 \\
\hline $220 / 0,5$ e revenida $400^{\circ} \mathrm{C} / 1 \mathrm{~h}$ & $5,9^{*}$ & $1,6^{*}$ & 47,1 & 1460 & 1614 & 11,0 & 8,3 \\
\hline $220 / 48$ & 9,5 & 1,1 & 47,1 & 1650 & 1974 & 17,8 & 2,5 \\
\hline $220 / 48$ e revenida $400^{\circ} \mathrm{C} / 1 \mathrm{~h}$ & $4,4^{*}$ & $1,4^{*}$ & 47,1 & 1493 & 1623 & 13,4 & 8,1 \\
\hline $270 / 0,5$ & 18,4 & 1,7 & 8,4 & 1273 & 1729 & 24,5 & 4,2 \\
\hline $270 / 0,5$ e revenida $400^{\circ} \mathrm{C} / 1 \mathrm{~h}$ & 6,4 & 1,3 & 8,4 & 1102 & 1346 & 11,2 & 9,3 \\
\hline $270 / 48$ & 15,2 & 1,6 & 8,4 & 1500 & 1762 & 21,1 & 3,5 \\
\hline $270 / 48$ e revenida $400^{\circ} \mathrm{C} / 1 \mathrm{~h}$ & $3,5^{*}$ & $1,0^{*}$ & 8,4 & 1236 & 1428 & 11,5 & 7,0 \\
\hline
\end{tabular}

*Valores não confiáveis devido à decomposição da austenita retida durante o revenimento.

Uma maior energia de impacto absorvida foi observada para as amostras tratadas a $270^{\circ} \mathrm{C}$ isso porque maior fração volumétrica de austenita retida e ferrita bainítica. Para as amostras revenidas pode ser observada uma menor energia de impacto absorvida e uma queda contínua dos valores de microdureza devido à decomposição da austenita retida.

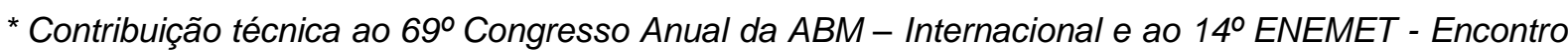
Nacional de Estudantes de Engenharia Metalúrgica, de Materiais e de Minas, 21 a 25 de julho de 2014, São Paulo, SP, Brasil.
} 


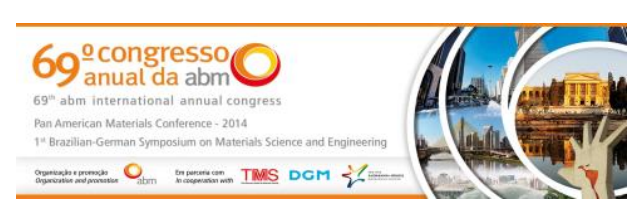

\section{CONCLUSÃo}

O estudo realizado no aço comercial SAE 9254 tratado isotermicamente em diferentes temperaturas abaixo de $\mathrm{Mi}_{\mathrm{i}}$ e posteriormente revenido resultaram nas seguintes conclusões:

- As amostras tratadas isotermicamente a 200,220 e $270^{\circ} \mathrm{C}$ apresentaram os constituintes martensita, ferrita bainítica e austenita retida. A amostra tratada nessa última temperatura apresentou uma maior quantidade de ferrita bainítica e austenita retida, conforme a tabela 2 e figura 2 .

- As amostras tratadas isotermicamente e revenidas apresentaram propriedades mecânicas de tração superiores às amostras apenas tratadas isotermicamente. No entanto, energia de impacto absorvida inferior às amostras tratadas isotermicamente devido a decomposição de austenita retida.

- Um grande efeito da fração volumétrica de austenita retida foi obervado nos resultados da energia de impacto absorvida e de microdureza. Observou-se que quanto maior a fração volumétrica de austenita retida, maior foi a energia de impacto absorvida, independente se esta austenita retida estava na forma de filmes ou de blocos.

\section{Agradecimentos}

Esse trabalho foi financiado pela Fundação de Amparo à Pesquisa do Estado de Minas Gerais (FAPEMIG), pela Coordenação de Aperfeiçoamento de Pessoal de Nível Superior (CAPES) e pelo Conselho Nacional de Desenvolvimento Científico e Tecnológico - CNPq.

\section{REFERÊNCIAS}

1 Honeycombe RWK, Bhadeshia HKDH. Steels Microstructure and Properties. $2^{\text {nd }}$ ed. Londres: Gray Publishing; 1995.

2 Takahashi M, Bhadeshia HKDH. Model for transition from upper to lower bainite. Materials Science and Technology. 1990;6:592-603.

3 Chang LC. Microstructures and reaction kinetics of bainite transformation in Si-rich steels. Materials Science and Engineering A. 2004;368:175-182.

4 Caballero FG, Bhadeshia HKDH, Mawella KJA, Jones DG, Brown P. Very strong low temperature bainite. Materials Science Technology. 2002;8:279-284.

5 Kozeschnik E, Bhadeshia HKDH. Influence of silicon on cementite precipitation in steels. Materials Science Technology. 2008;24:343-347.

6 Bhadeshia HKDH, Edmonds DV. The Bainite Transformation in a Silicon Steel. Metallurgical and Materials Transaction A. 1979;10:895-907.

7 Leslie WC. The physical metallurgy of steels. Nova lorque: Mc Graw-Hill; 1982.

8 Bhadeshia HKDH, Edmonds DV. Bainite in silicon steels: new composition-property approach, part 1. Metals Science. 1983;17:411-419.

9 Bhadeshia HKDH, Edmonds DV. Bainite in silicon steels: new composition-property approach, part 2. Metals Science. 1983;17:420-425.

10 Bhadeshia HKDH, Waugh AR. Bainite: an atom-probe study of the incomplete reaction phenomenon. Acta Metallurgica. 1982;30:775-784.

11 Chang LC, Bhadeshia HKDH. Carbon content of austenite in isothermally transformed 300M Steel. Materials Science and Engineering A. 1994;184:L17-L19.

\footnotetext{
* Contribuição técnica ao $69^{\circ}$ Congresso Anual da ABM - Internacional e ao 14ํㅡㄹ ENEMET - Encontro Nacional de Estudantes de Engenharia Metalúrgica, de Materiais e de Minas, 21 a 25 de julho de 2014, São Paulo, SP, Brasil.
} 


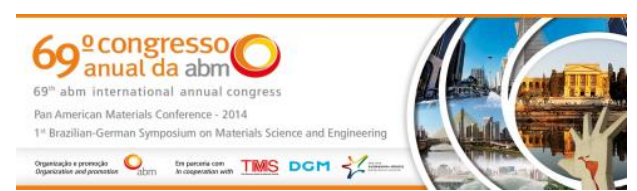

12 Caballero FG, Bhadeshia HKDH. Very strong bainite - current opinion in solid state. Materials Science. 2004;8:251-257.

13 Garcia-Mateo C, Caballero FG, Bhadeshia HKDH. Development of hard bainite. ISIJ International. 2003;43:1238-43.

14 Garcia-Mateo C, Caballero FG, Bhadeshia HKDH. Superbainita, una nueva microestructura bainítica de alta resistência. Revista Metallurgy Madrid. 2005;41:186193.

15 Caballero FG, Chao J, Cornide J, García-Mateo C, Santofimia MJ, Capdevila C. Toughness deterioration in advanced high strength bainitic steels. Materials Science and Engineering A. 2009;525:87-95.

16 Garcia-Mateo C, Caballero FG, Chao J, Capdevila C, Andrés GC. Mechanical Stability of retained austenite during plastic deformation of super high strength carbide free bainitic steels. Journal of Materials Science. 2009;44:4617-24.

17 Caballero FG, García-Mateo C, Chao J, Santofimia MJ, Capdevila C, Andrés GC. Effects of morphology and stability of retained austenite on the ductility of TRIP-aided bainitic steels. ISIJ International. 2008;48:1256-62.

18 Caballero FG, Santofimia MJ, Capdevila C, García-Mateo C, García AC. Design of advanced bainitic steels by optimisation of TTT diagrams and $T_{0}$ curves. ISIJ International. 2006;46:1479-88.

19 Khare S, Lee K, Bhadeshia HKDH. Carbide-free bainite: compromise between rate of transformation and properties. Metallurgical Materials Transaction A. 2010;41:922-928.

20 Peet MJ, Hill P, Rawson M, Wood S, Bhadeshia HKDH. Fatigue of extremely fine bainite. Materials Science and Technoogy - London. 2011;27:119-123.

21 Zhang FC, Wang TS, Zhang P, Zhang CL, Lv B, Zhang M, Zhang, YZA. Novel method for the development of a low-temperature bainitic microstructure in the surface layer of low-carbon steel. Scripta Materialia. 2008;59:294-296.

22 Menapace C, Lonardelli I, Tait M, Moinari A. Nanostrucutred/ultrafine multiphase steel with enhanced ductility obtained by mechanical alloying and spark plasma sintering of powders. Materials Science and Engineering A. 2009;517:2009.

23 American Society for Testing and Materials. ASTM E8: Standard test methods of tension testing of metallic materials. West Conshohocken; 2008.

24 American Society for Testing and Materials. ASTM E23: Standard test methods for notched bar impact testing of metallic materials. West Conshohocken; 2012.

25 Radcliffe S, Rollason E. The kinetics of the formation of bainite in high-purity iron-carbon alloys. Journal of the Iron and Steel Institute. 1959;191:56-65.

26 Van Bohemen SMC, Santofimia MJ, Sietsma J. Experimental evidence for bainite formation below Ms in Fe-0.66C. Scripta. Materialia. 2008;58:488-91.

27 Kolmskog P, Borgenstam A, Hillert M, Hedström P, Babu SS, Terasaki H, Komizo Y. Direct observation that bainite can grow below Ms. Metallurgical and Materials Transactions A. 2012;43A:4984-88.

28 Steven W, Haynes AG. The temperature of formation of martensite and bainite in lowalloy steels. Journal of the Iron and Steel Institute. 1956;183:349-356.

29 Junior JAC, Santos DB. Effect of tempering temperature on isothermal decomposition product formed below Ms. Journal of Materials Research and Technology. 2013;2:93-99.

30 Koinstinen DP, Marburger RE. A general equation prescribing the extent of the austenite-martensite transformation in pure iron-carbon alloys and plain carbon steels. Acta Metallurgica. 1959;7:59-60.

31 Cullity BD, Stock SR. Elements of x-ray diffraction. $3^{\underline{a}}$ ed. Nova lorque: Addison- Wesley Publishing Co., Inc.; 2001.

32 Dyson DJ, Holmes B. Effect of alloying additions on the lattice parameter of austenite. Journal of the Iron and Steels Institute. 1970;208:469-474.

33 Saha Podder A, Bhadeshia HKDH. Thermal stability of austenite retained in bainitic steels. Materials Science and Engineering A. 2010;527:2121-28.

\footnotetext{
* Contribuição técnica ao $69^{\circ}$ Congresso Anual da ABM - Internacional e ao 14ํㅡㄹ ENEMET - Encontro Nacional de Estudantes de Engenharia Metalúrgica, de Materiais e de Minas, 21 a 25 de julho de 2014, São Paulo, SP, Brasil.
} 
